\title{
LVIII. On a new optical property of biaxial crystals
}

\section{C.V. Raman M.A. \& V.S. Tamma M.Sc.}

To cite this article: C.V. Raman M.A. \& V.S. Tamma M.Sc. (1922) LVIII. On a new optical property of biaxial crystals, Philosophical Magazine Series 6, 43:255, 510-513, DOI: 10.1080/14786442208633904

To link to this article: http://dx.doi.org/10.1080/14786442208633904

\section{Published online: 08 Apr 2009.}

6 Submit your article to this journal $x$

\section{山 Article views: 2}

Q View related articles

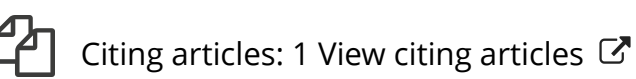




\section{Prof. C. V. Raman and Mr. V. S. Tamma on a}

be omitted, i.e. if $k$ be zero, the amplitude graph will become a strict parabola. There will be no upper limit to the value of the amplitude, and therefore the collapse of maintenance will not be explained. If the frictional term involving the first power of the velocity be omitted, i. e. if $k^{\prime}$ be zero, there will be no explanation for the fact that there is a lower limit to the value of $\alpha$ below which there is no maintenance. Facts (5) and (7) will be left unexplained. With the two frictional terms all the facts are indicated.

University College, Rangoon.

May 10, 1921.

I.VIII. On a New Optical Property of Biaxial Crystals. By (. V. Raman, M.A., Palit Professor of Physits in the Calcutta University, and V. S. TAMma, M.Sc., Lecturer in Physics, Meerut College, India *

\section{Introduction.}

1 FACT of singular interest and importance in the optics A of crystalline media which appears hitherto to have been overlooked is that a plate of a biaxial crystal bounded by parallel faces is capable of tocussing divergent rays proceeding from in distant light-source and forming real images of it. Very simple apparatus will suffice to observe this phenomenon. The incandescent filament of a tiny 2-volt lamp or an illuminated pin-hole serves as a suitable source of light. At some distance from it is placed a crystal of aragonite cut and polished with parallel-faces at right angles to the bisectrix of the acute angle between the optic axes. On suitably orienting the crystal and examining the pencil of light which has passed through it, a real erect unpoliarized image of the luminous filament may easily be picked up and traced continuously a way from the crystal for a considerable distance. There is no difficulty in receiving and observing the image directly on a plate of ground glass if desired.

The image is sharp and bright and practically achromatic if the object and the place of observation are both within a few centimetres of the crystal, one on each side. As either the object or the place of observation is drawn away from the crystal the image spreads out into a spectrum. Using monochromatic light, however, it can be seen that the image remains quite well defined and sufficiently bright to be

\footnotetext{
* Communicated by Prof. A. W. Porter, F.R.S.
} 
traced for a distance of some 30 or $40 \mathrm{~cm}$. from the crystal. The effects are specially beautiful if observed in the light of a mercury vapour lamp. Separate images of the pinhole corresponding to the yellow, green, and violet radiations of the mercury vapour may be seen, and images corresponding to the fainter components of the line spectrum may also be observed by cutting out the superfuous light with suitable colour filters. A simple slit with a plate of aragonite crystal and an eye-lens to observe the image thus functions as a little spectroscope, which has quite a marked dispersion in the region of shorter wave-lengths.

It should be remarked that these optical images formed by an aragonite plate differ from those formed by an ordinary converging lens in several respects. 'The images in the present case are real, erect, and of unit magnitication irrespective of the distance of either objert or image from the crystal. Further, the image is continuous, that is, it may be observed anywhere in the prolongation of a certain line for a considerable distance from the crystal and not merely at a single point as in the case of the images formed by a lens. Also the images appear-sharply defined in a field of diffuse light, showing that only part of the energy passing through the crystal is brought to a focus. The olject being fixed, the image moves when the orientation of the erystal is altered, but not when the plate is moved in its oun plane. The focussing property, in other words, appears to be related to a fixed direction within the crystal. In order that the image may be within the field of observation it is necessary, in fact, that the bundle of light-rays should pass through the crystal roughly in this fixed direction, which appears to be that of either axis of single ray velocity in the crystal.

\section{Explanation of the Phenomenon.}

'The fact last mentioned suggests a mode of approach to a theoretical explanation of the phenomenon. As already mentioned, the object may be placed at a distance from the crystal, but for simplicity we shall first consider the case in which the object is a point source of light placed just on one of its surfuces. The form of the wave-fronts in the disturbance diverging within the crystal is the Fresnel surfact of two sherts which, as is well known, has a singular point in the direction of either axis ol single-ray velocity. It we resolve the wave-front into the gronp of plane waves of "hich it is the envelope, we see at once that the singularity is really the crossing point of an infinite number of pline waves the 
normals to which generate the surface of a cone, and hence the intensity of the disturbance at the singular point must be very great in comparison with that at other points on the wave. On emergence from the crystal the singularity persists, and, since in its immediate neighbourhood the wavefront is approximately symmetrical in shape about the axis of the cone of normals, it can easily be seen that the advancing front would retain the same general configuration exhibiting a singularity or concentration of luminosity along: its course, as indicated in fig. 1.

Fig. 1.

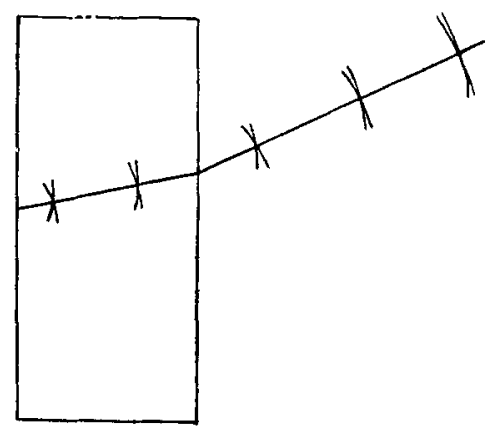

We have thus in effect a continuous image of the source along a line. When the source instead of being placed on the surface is removed to a distance from the crystal, the waves which diverge from it are in the first instance spherical, but on entry into the crystal these divide at once into two sheets, the points of intersections of which must be in the nature of foci or concentrations of luminosity in the wave-front. On emergence of the waves from the crystal the same effect is propagated outwards, giving a cortinuous focus or image of the source in much the same way as in the case illustrated in fig. 1 . When the luminous source is of finite dimensions we have an jmage corresponding to each point of it, and it is easy to see that we should have a complete picture built up which would be of the same dimensions as the source and similarly oriented.

The spectral dispersion of the image is also easily understood. For, the direction in which the singularity travels within the crystal being inclined to the normal to the plate, the direction in which the singularity travels on emergence 
from the crystal depends on the refractive index and hence inust make a greater angle with the normal to the plate for the shorter wave-lengths. This is exactly what is actually observed. In an exact quantitative discussion the fact that the direction of the axis of single-ray velocity in the crystal varies with the wave-length must also be taken into account. A fuller discussion of this point and of the resolving power of the crystalline plate regarded as a spectroscope in itself would be of interest. This would obviously require a determination of the distribution of luminosity in and around the point of singularity in the wave-front. It is hoped at an early opportunity to carry out a detailed investigation on these points.

\section{Summary and Conclusion.}

(a) The paper describes a new optical effect observed with a biaxial crystalline plate, viz., that such a plate though bounded by parallel faces, is capable of forming on one side of it when suitably oriented a real erect image of a source of light such as a luminous filament placed at some distance from it on the other side.

(b) The characteristics of this image-forming property are set ont in detail, a noteworthy feature being the spectral resolution of the image which occurs when either the object or the image is at a considerable distance from the crystal.

(c) A theoretical explanation of the phenomenon is suggested, viz., that the singular point in the Fresnel wavesurface is a locus of maximum intensity, and, owing to the fact that the wave-form on emergence from the crystal retains its general character, we get a continuous concentration of luminosity along a line any point of which is in effect an optical image of the source.

The investigation described in this paper was carried out in part in the senior author's laboratory at Calcutta, and in part at University College, London, during the visit to England of the senior author, who wishes to express his cordial thanks to Prof. A. W. Porter, F.R.S., for his hospitality and kind interest in providing the necessary facilities for the work. 